\title{
Moments of the Auto-Correlation Function and the KMS-Condition
}

\author{
M. Fannes`, R. Martens, and A. Verbeure \\ Instituut voor Theoretische Fysica, Universiteit Leuven, B-3030 Leuven, Belgium
}

\begin{abstract}
Inequalities between successive moments of the time dependent auto-correlation function are derived. Furthermore, we prove that they provide an infinite set of characterizations of an equilibrium state.
\end{abstract}

\section{Introduction}

As is well known, the time auto-correlation function is an important quantity for the study of macroscopic systems both from a theoretical as well as from an experimental point of view. In particular we have in mind response theory. In practice, computations in this theory are generally stopped after the first moment because of lack of information about the higher moments of the autocorrelation function. If this is the case, one speaks about linear response theory [1-4]. Indeed it is not always easy to establish a radius of convergence for this auto-correlation function expressed as a power series in the time variable [5]. Motivated by this problem, we study in a rigorous way the relation between successive terms of this series expansion for KMS-states and derive inequalities between them (see Theorem II.4 and 5). Furthermore we prove that our inequalities are best possible in the sense that each of them characterizes equilibrium or KMS-states (see Theorem III.2 and 3).

First we introduce the scheme in which we work. Let $\mathscr{M}$ be a von Neumann algebra on a Hilbert space $\mathscr{H}$ and $H$ a self-adjoint operator on $\mathscr{H}$. Let $U_{t}=\exp i t H, t \in \mathbb{R}$ such that $t \rightarrow \alpha_{t}=U_{t} \cdot U_{t}^{*}$ is a continuous one parameter group of *-automorphisms of $\mathscr{M}$. Let $\Omega$ be a normalized element of $\mathscr{H}$ cyclic for $\mathscr{M}$. We denote by $\omega$ the vector state determined by $\Omega$.

For any $f \in \mathscr{L}^{1}(\mathbb{R})$, denote by $\hat{f}$ the Fourier transform $\int d x f(x) e^{i k x}=\hat{f}(k)$ of $f$. Denote by $\mathscr{M}_{0}$ the algebra generated by the set

$$
\left\{x(f) \in \mathscr{M} \mid x(f)=\int d t f(t) \alpha_{t} x, x \in \mathscr{M}, \hat{f} \in \mathscr{D}\right\},
$$

* Bevoegdverklaard Navorser NFWO, Belgium 
where $\mathscr{D}$ is the set of $C^{\infty}$-functions with compact support on $\mathbb{R}$. Remark that $\mathscr{M}_{0}$ is a strongly dense ${ }^{*}$-subalgebra of $\mathscr{M}$. We define the derivation $L$ on $\mathscr{M}_{0}$ by

$$
L^{n} x(f)=i^{n} x\left(f^{(n)}\right) \quad n=1,2, \ldots
$$

We define the symmetrized $n^{\text {th }}$ order moment of the auto-correlation function as: for $x \in \mathscr{M}_{0}$

$$
m_{n}(x)=\left(x \Omega, L^{n} x \Omega\right)+\left(x^{*} \Omega, L^{n} x^{*} \Omega\right),
$$

for $n=0,1,2, \ldots$.

\section{Inequalities for Moments of an Equilibrium State}

In this section we derive correlation inequalities for the symmetrized moments (2) when the state $\omega$ is an equilibrium state or KMS-state for the evolution $\alpha_{t}$ determined by the derivation $L$.

Definition II.1. The state $\omega$ of $\mathscr{M}$ is called a KMS-state at inverse temperature $\beta=1$ if it satisfies the following condition: the set $\mathscr{M} \Omega$ belongs to the domain $\mathscr{D}\left(\Delta^{1 / 2}\right)$, where $\Delta=\exp (-H)$, and for all $x, y \in \mathscr{M}$ we have

(i) $H \Omega=0$

(ii) $\left(\Delta^{1 / 2} x \Omega, \Delta^{1 / 2} y \Omega\right)=\left(y^{*} \Omega, x^{*} \Omega\right)$.

First we derive an integral representation for the moments.

Lemma II.2. If $\omega$ satisfies the KMS-condition, then for each $x \in \mathscr{M}_{0}, x \neq 0$, there exists a probability measure $\mu_{x}$ on $\mathbb{R}^{+}=[0, \infty)$ such that

where

$$
\frac{m_{n}(x)}{m_{0}(x)}=\int_{\mathbb{R}^{+}} f_{n}(\lambda) d \mu_{x}(\lambda), \quad n=0,1,2, \ldots,
$$

$$
\begin{aligned}
f_{2 n}(\lambda) & =\lambda^{2 n} ; \\
f_{2 n+1}(\lambda) & =\lambda^{2 n+1} \text { th } \lambda / 2, \quad \lambda \in \mathbb{R}^{+} .
\end{aligned}
$$

Furthermore, let $f_{-1}(\lambda)=\frac{\text { th } \lambda / 2}{\lambda}, \lambda \in \mathbb{R}^{+}$. If $m_{-1}(x)$ is defined by

$$
\frac{m_{-1}(x)}{m_{0}(x)}=\int_{\mathbb{R}^{+}} f_{-1}(\lambda) d \mu_{x}(\lambda)
$$

then the Duhamel two-point function

$$
(x, x)_{\sim}=\int_{0}^{1} d t\left\|\Delta^{t / 2} x \Omega\right\|^{2},
$$

satisfies $(x, x)_{\sim}=m_{-1}(x)$.

Proof. Let $H=\int \lambda d E_{\lambda}$ be the spectral resolution of $H$ on $\mathscr{H}$. By the KMScondition:

$$
d\left\|E_{-\lambda} x^{*} \Omega\right\|^{2}=e^{-\lambda} d\left\|E_{\lambda} x \Omega\right\|^{2}, \quad x \in \mathscr{M} .
$$


Define the probability measure $\mu_{x}$ on $\mathbb{R}^{+}$, for $x \neq 0$ :

$$
d \mu_{x}(\lambda)=\frac{\left(1+e^{-\lambda}\right) d\left(\left\|E_{\lambda} x \Omega\right\|^{2}+\left\|E_{\lambda} x^{*} \Omega\right\|^{2}\right)}{\left\|x^{*} \Omega\right\|^{2}+\|x \Omega\|^{2}},
$$

which is well defined as $\omega$ is separating. Formulae (3) follow by a straightforward computation and the remark that

$$
L^{n}(x) \Omega=H^{n} x \Omega, \quad x \in \mathscr{M}_{0} .
$$

To prove (4), compute

$$
m_{-1}(x)=\int_{\mathbb{R}^{+}} \frac{1-e^{-\lambda}}{\lambda} d\left(\left\|E_{\lambda} x \Omega\right\|^{2}+\left\|E_{\lambda} x^{*} \Omega\right\|^{2}\right) .
$$

Using again the KMS-condition

$$
m_{-1}(x)=\int_{\mathbb{R}} \frac{1-e^{-\lambda}}{\lambda} d\left\|E_{\lambda} x \Omega\right\|^{2}=(x, x)_{\sim} .
$$

Lemma II.3. The functions $F_{n}: \mathbb{R}^{+} \rightarrow \mathbb{R}^{+}(n=1,2, \ldots)$ given $b y$

(i) $F_{2 n}\left(y^{2 n}\right)=y^{2 n+1}$ th $y$

(ii) $F_{2 n-1}\left(y^{2 n-1}\right.$ th $\left.y\right)=y^{2 n}$,

and the function $F:(0,1] \rightarrow \mathbb{R}^{+}$given by $F\left(\frac{\text { th } y}{y}\right)=y$ th $y$, are well defined and convex.

Proof. (i) Check that

$$
(2 n)^{2} y^{2 n-1} F_{2 n}^{\prime \prime}\left(y^{2 n}\right)=(2 n+1) \text { th } y+(2 n+3) y / \operatorname{ch}^{2} y-2 y^{2} \operatorname{sh} y / \operatorname{ch}^{3} y,
$$

and remark that

$$
\operatorname{ch} y=1+\int_{0}^{y} \operatorname{sh} t d t \geqq 1+\int_{0}^{y} t d t=1+\frac{y^{2}}{2} \geqq y .
$$

Inserting this inequality in the last term of $(*)$, yields $F_{2 n}^{\prime \prime} \geqq 0$ for all $n \geqq 1$.

To prove (ii) we use the following characterization of convexity [6, Appendix I, Theorem A.2]:

$$
\operatorname{det}\left|\begin{array}{lll}
1 & a_{1} & F_{2 n-1}\left(a_{1}\right) \\
1 & a_{2} & F_{2 n-1}\left(a_{2}\right) \\
1 & a_{3} & F_{2 n-1}\left(a_{3}\right)
\end{array}\right| \geqq 0
$$

for $0 \leqq a_{1} \leqq a_{2} \leqq a_{3}$. Taking

$$
a_{i}=y_{i}^{2 n-1} \text { th } y_{i} ; \quad i=1,2,3
$$

and using the monotonicity of the function

$$
y \rightarrow y^{2 n-1} \text { th } y \quad \text { on } \mathbb{R}^{+},
$$


the condition $(* *)$ becomes, after interchanging two columns,

$$
\operatorname{det}\left|\begin{array}{lll}
1 & y_{1}^{2 n} & y_{1}^{2 n-1} \text { th } y_{1} \\
1 & y_{2}^{2 n} & y_{2}^{2 n-1} \text { th } y_{2} \\
1 & y_{3}^{2 n} & y_{3}^{2 n-1} \text { th } y_{3}
\end{array}\right| \leqq 0
$$

for $0 \leqq y_{1} \leqq y_{2} \leqq y_{3}$, which is equivalent to the concavity of the function

Compute:

$$
G_{2 n-1}\left(y^{2 n}\right)=y^{2 n-1} \text { th } y .
$$

$$
y^{2 n+1}(2 n)^{2} \operatorname{ch}^{2} y G_{2 n-1}^{\prime \prime}\left(y^{2 n}\right)=(2 n-1)(y-\operatorname{sh} y \operatorname{ch} y)-2 y^{2} \text { th } y \leqq 0,
$$

as $y \leqq \operatorname{sh} y \operatorname{ch} y$. Finally the convexity of the function $F$ given by $F\left(y^{-1}\right.$ th $\left.y\right)$ $=y$ th $y$ is easily seen by computing its second derivative:

$$
F^{\prime \prime}\left(\frac{\text { th } y}{y}\right)=\frac{2 y^{3}\left(2 y^{2}-y \operatorname{th} y-\operatorname{sh}^{2} y\right) \operatorname{ch}^{4} y}{(y-\operatorname{sh} y \operatorname{ch} y)^{3}} .
$$

It is sufficient to prove that

$$
y \rightarrow f(y)=2 y^{2}-y \text { th } y-\operatorname{sh}^{2} y
$$

is negative. However

$$
f(y)=\int_{0}^{y} d x \frac{1}{\operatorname{ch}^{2} x} \int_{0}^{x} d z 8(z-\operatorname{ch} z \operatorname{sh} z) \operatorname{ch} z \operatorname{sh} z
$$

In the follwing theorem we derive inequalities between successive moments.

Theorem II.4. If $\omega$ is a KMS-state, we have, with the notations of above, for $x \in \mathscr{M}_{0}$ and $x \neq 0$ :

a) $F\left(\frac{2 m_{-1}(x)}{m_{0}(x)}\right) \leqq \frac{1}{2} \frac{m_{1}(x)}{m_{0}(x)}$

b) for $n=1,2, \ldots$

$$
\begin{aligned}
F_{2 n}\left(\frac{m_{2 n}(x)}{2^{2 n} m_{0}(x)}\right) & \leqq \frac{m_{2 n+1}(x)}{2^{2 n+1} m_{0}(x)} \\
F_{2 n-1}\left(\frac{m_{2 n-1}(x)}{2^{2 n-1} m_{0}(x)}\right) & \leqq \frac{m_{2 n}(x)}{2^{2 n} m_{0}(x)} .
\end{aligned}
$$

Proof. The result of the theorem is now an immediate consequence of Jenssens inequality applied to the representation of the $m_{n}(x)$ obtained in Lemma II.2 using the convex functions $F$ and $F_{n}(n=1,2, \ldots)$ of Lemma II.3.

Remark that the inequality (5) has been obtained before by Falk and Bruch [7], the special case of inequality (7) with $n=1$ was first derived by Martens and Verbeure [8]. The other inequalities relating higher moments are new as far as we know. 
Furthermore from the formulation and the proof of Theorem II.4 it looks as if the inequalities (5), (6) and (7) are different in nature. We write all the inequalities in a more organized way and obtain a chain of inequalities such that all of them are of the same nature. Moreover this way of writing enables us to complete the series with the upper bound of the Duhamel two-point function, as derived by Roepstorff [9].

In order to do this, use the monotonicity of the functions $f_{n}(n=$ $-1,1,2,3, \ldots)$ given in Lemma II.2 to define the (positive) means $M_{n}(x)(n=$ $-1,1,2,3, \ldots)$ of the moments by

$$
f_{n}\left(2 M_{n}(x)\right)=\frac{m_{n}(x)}{m_{0}(x)} .
$$

Furthermore define the mean $N_{0}(x)$ by

$$
\text { th } N_{0}(x)=\left|\frac{\omega\left(\left[x, x^{*}\right]\right)}{m_{0}(x)}\right| .
$$

Theorem II.5. If $\omega$ is a KMS-state, with the notations of above, we have for all $x \in \mathscr{M}_{0}, x \neq 0$ :

$$
N_{0}(x) \leqq M_{-1}(x) \leqq M_{1}(x) \leqq M_{2}(x) \leqq \ldots \leqq M_{n}(x) \leqq \ldots
$$

Proof. First we check that $N_{0}(x) \leqq M_{-1}(x)$ is equivalent to the known upper bound for the Duhamel two-point function:

$$
\begin{array}{rlrl}
(x, x)_{\sim} & \leqq \frac{\omega\left(\left[x, x^{*}\right]\right)}{\ln \frac{\omega\left(x x^{*}\right)}{\omega\left(x^{*} x\right)}} & & \text { if } \omega\left(\left[x, x^{*}\right]\right) \neq 0 \\
& \leqq \frac{1}{2} \omega\left(x x^{*}+x^{*} x\right) & \text { if } \omega\left(\left[x, x^{*}\right]\right)=0 .
\end{array}
$$

Indeed, this inequality can be written as

$$
\frac{(x, x)_{\sim}}{2^{-1} m_{0}(x)} \leqq \frac{\frac{\omega\left(\left[x, x^{*}\right]\right)}{m_{0}(x)}}{\frac{1}{2} \ln \frac{\omega\left(x x^{*}\right)}{\omega\left(x^{*} x\right)}}, \quad \text { if } \quad \omega\left(\left[x, x^{*}\right]\right) \neq 0,
$$

or in the notation of (8) and (9)

$$
\frac{\operatorname{th} M_{-1}(x)}{M_{-1}(x)} \leqq \frac{\text { th } N_{0}(x)}{N_{0}(x)}
$$

where we used the formula

$$
\operatorname{arcth} r=\log \left(\frac{1+r}{1-r}\right)^{1 / 2} \quad \text { if } 0 \leqq r<1
$$

The case $\omega\left(\left[x, x^{*}\right]\right)=0$ is immediate. The equivalence follows by remarking that the function $x \in \mathbb{R}^{+} \rightarrow \frac{\operatorname{th} x}{x}$ is monotonically decreasing. 
Now we prove that $M_{-1}(x) \leqq M_{1}(x)$ is equivalent with inequality (5). In the notation of (8), the inequality (5) reads as

$$
M_{1}(x) \text { th } M_{1}(x) \geqq F\left(\frac{\operatorname{th} M_{-1}(x)}{M_{-1}(x)}\right)=M_{-1}(x) \text { th } M_{-1}(x) .
$$

The equivalence follows from the remark that the function $x \in \mathbb{R}^{+} \rightarrow x$ th $x$ is monotonically increasing. Furthermore, we prove that $M_{2 n}(x) \leqq M_{2 n+1}(x)$ (n $=1,2, \ldots)$ is equivalent with inequalities (6). In the notation of (8) inequalities (6) read:

$$
M_{2 n+1}(x)^{2 n+1} \text { th } M_{2 n+1}(x) \geqq F_{2 n}\left(M_{2 n}(x)^{2 n}\right)=M_{2 n}(x)^{2 n+1} \text { th } M_{2 n}(x),
$$

or $M_{2 n}(x) \leqq M_{2 n+1}(x)$ by the monotonicity of $x \rightarrow x^{2 n+1}$ th $x$ on $\mathbb{R}^{+}$. Finally $M_{2 n-1}(x) \leqq M_{2 n}(n=1,2, \ldots)$ is equivalent with inequalities (7):

$$
\left.M_{2 n}(x)^{2 n} \geqq F_{2 n-1}(x)^{2 n-1} \text { th } M_{2 n-1}(x)\right)=M_{2 n-1}(x)^{2 n},
$$

written in the notation of (8) or $M_{2 n}(x) \geqq M_{2 n-1}(x)$. Combining all these inequalities we get the chain (10).

This theorem yields an infinite set of inequalities between the consecutive moments of the autocorrelation function in such a way that each inequality contains only two normalized moments. It is clear that this result is important to study the properties of the time dependent autocorrelation function (see e.g. [5]). But instead of proceeding to applications, we want to examine in the next section the question: how good are these inequalities? In fact we prove that they are the best possible ones in the sense that it is sufficient to impose inequality (5) or inequalities (6) and (7) for a single value of $n$ in order to characterize a KMS-state.

\section{Characterizations of Equilibrium States}

We start with the set up outlined in the introduction; $\mathscr{M}$ is a von Neumann algebra acting on a Hilbert space $\mathscr{H}$ with normalized cyclic vector $\Omega ; H$ is a self-adjoint operator on $\mathscr{H}$ defining the one-parameter group $\left(\alpha_{t}\right)_{t \in \mathbb{R}}$ of automorphisms of $\mathscr{M}$. The derivation $L$ is defined on $\mathscr{M}_{0}$ in (1) and the momenta $m_{n}(x), n=1,2, \ldots, x \in \mathscr{M}_{0}$ in (2). Finally we define for $x \in \mathscr{M}_{0}$

$$
m_{-1}(x)=\int_{0}^{1} d s\left(x \Omega, \alpha_{i s}(x) \Omega\right) .
$$

In the case of a KMS-state this definition coincides with the one in Lemma II. 2 .

First we prove that if the odd order moments are real valued then the state $\omega(\cdot)=(\Omega, \cdot \Omega)$ of $\mathscr{M}$ is $\alpha_{t}$-invariant.

Lemma III.1. Let $n \in \mathbb{N}$, then $m_{2 n-1}(x)$ real for all $x \in \mathscr{M}_{0}$ implies that $\omega\left(\alpha_{t}(x)\right)$ $=\omega(x)$ for all $x \in \mathscr{M}$. 
Proof. First we treat the case $n=0$. Take $x=x^{*} \in \mathscr{M}_{0}$. The reality of $m_{-1}(x)$ implies

$$
\int_{0}^{1} d s\left\{\left(\Omega, \alpha_{-i s}(x) x \Omega\right)-\left(\Omega, x \alpha_{i s}(x) \Omega\right)\right\}=0 .
$$

Replacing $x$ by $1+x$ yields

$$
\int_{0}^{1} d s\left\{\left(\Omega, \alpha_{-i s}(x) \Omega\right)-\left(\Omega, \alpha_{i s}(x) \Omega\right)\right\}=0 .
$$

Replacing $x$ by $L x$ yields

$$
(\Omega,(\operatorname{ch} L-1) \times \Omega)=0
$$

implying $(\Omega, L(x) \Omega)=0$, by functional calculus. If $n=1,2, \ldots$, take again $x$ $=x^{*} \in \mathscr{M}_{0}$, then the reality implies

$$
\left(\Omega, x L^{2 n-1}(x) \Omega\right)+\left(\Omega, L^{2 n-1}(x) x \Omega\right)=0 .
$$

Again replacing $x$ by $1+x$ yields

$$
\left(\Omega, L^{2 n-1}(x) \Omega\right)=0
$$

implying again $(\Omega, L(x) \Omega)=0$.

Now we prove the main results of this section. In the following we use the notation introduced above and extend the definitions of $M_{-1}(x)$ and $N_{0}(x)$ (see (8), (9)) to the limit cases $m_{-1}(x)=0$ and $\left|\left(\left[x, x^{*}\right]\right)\right|=m_{0}(x)$.

Theorem III.2. Let $\mathscr{N}_{0}=\left\{x \in \mathscr{M}_{0} \mid m_{0}(x)>0\right\}$. If for all $x \in \mathscr{N}_{0}$ one of the following conditions holds:

(i) $m_{-1}(x) \geqq 0$ and $N_{0}(x) \leqq M_{-1}(x)$

or (ii) $m_{-1}(x) \geqq 0, m_{1}(x) \geqq 0$ and $M_{-1}(x) \leqq M_{1}(x)$,

where $M_{-1}(x)$ and $M_{1}(x)$ are defined in (8) and $N_{0}(x)$ in (9), then the state $\omega$ is a KMS-state.

In the next theorem we give another infinite set of equilibrium conditions. In Theorem III.2 there are no other conditions to impose on the dynamical system except for the inequalities. For the other inequalities of Theorem II.5 to determine a KMS-state, it is clear that a supplementary condition is needed in general because they become trivial in the case that $H=0$. As in our notation $\beta$ is always absorbed in $H$, this would also correspond to the exceptional situation that $\beta=0$ or $T=\infty$. We impose a condition on the evolution to eliminate the degeneracies of the Hamiltonian, namely:

$$
\mathscr{M}_{\alpha}=\left\{x \in \mathscr{M} \mid \alpha_{t}(x)=x, t \in \mathbb{R}\right\}
$$

is abelian.

Remark that this condition is much weaker and of a completely different nature than ergodicity or clustering which are nonlinear conditions on the state often used in characterizations of equilibrium states [10-11]. 
Theorem III.3. Let $\mathscr{N}_{0}=\left\{x \in \mathscr{M}_{0} \mid m_{0}(x)>0\right\}$. If the evolution satisfies condition (12) and if for a fixed $n=1,2, \ldots$ and for all $x \in \mathscr{N}_{0}$ one of the following conditions holds

(i): $m_{2 n-1}(x) \geqq 0, m_{2 n+1}(x) \geqq 0 \quad$ and $\quad M_{2 n-1}(x) \leqq M_{2 n}(x) \leqq M_{2 n+1}(x)$, or (ii): $m_{2 n-1}(x) \geqq 0$ and $M_{2 n}(x) \leqq M_{2 n+1}(x) \leqq M_{2 n+2}(x)$, where $M_{n}(x)(n=1,2, \ldots)$ is defined in (8), then the state $\omega$ is a KMS-state.

The proofs of Theorems III.2 and III.3 will be performed in a number of steps.

Lemma III.4. Condition (i) of Theorem III.2 implies that $\omega$ is a KMS-state.

Proof. The time invariance of $\omega$ follows from Lemma III.1. If $x \in \mathscr{N}_{0}$, the proof of Theorem II.5 shows that condition (i) is equivalent to the upper bound of the Duhamel two point function

$$
\begin{aligned}
(x, x)_{\sim} & \leqq \frac{\omega\left(\left[x, x^{*}\right]\right)}{\ln \frac{\omega\left(x x^{*}\right)}{\omega\left(x^{*} x\right)}}, & & \text { if } \omega\left(\left[x, x^{*}\right]\right) \neq 0 \\
& \leqq \frac{1}{2} \omega\left(x x^{*}+x^{*} x\right), & & \text { if } \omega\left(\left[x, x^{*}\right]\right)=0 .
\end{aligned}
$$

On the other hand if $m_{0}(x)=0$, then $(x, x)_{\sim}=0$ by Schwartz inequality and the inequality remains valid.

The rest of the proof follows from [12, Theorem II.4].

Lemma III.5. Any state satisfying condition (ii) of Theorem III.2 or conditions (i) or (ii) of Theorem III.3 is separating.

Proof. Let $x \in \mathscr{M}$ such that $\omega\left(x x^{*}\right)=0$. It is sufficient to prove that $\omega\left(x^{*} x\right)=0$. Indeed then for all $y \in \mathscr{M}$ :

$$
y x^{*} \Omega \text { implies } x y^{*} \Omega=0,
$$

and as $\Omega$ is cyclic, $x=0$. Now we prove this by contradiction. Therefore let $\omega\left(x^{*} x\right)>0$. As $\omega$ is time invariant (Lemma III.1) there exists a self-adjoint operator which we again denote by $H$ and such that

$$
\alpha_{t}(x) \Omega=e^{i t H} x \Omega .
$$

Let $H=\int \lambda d E_{\lambda}$ be the spectral resolution of $H$ and $\widehat{f} \in \mathscr{D}$, then

and

$$
x_{f}=\int f(t) \alpha_{t}(x) d t \in \mathscr{M}_{0}
$$

$$
\omega\left(x_{f}\left(x_{f}\right)^{*}\right)=0
$$

because for all $t_{1}$ and $t_{2}$ :

$$
\omega\left(\alpha_{t_{1}}(x) \alpha_{t_{2}}\left(x^{*}\right)\right)=0
$$

by time invariance and Schwartz inequality. As $\omega\left(x^{*} x\right)>0$ there exists an $\tilde{f} \in \mathscr{D}$ such that $\omega\left(\left(x_{f}\right)^{*} x_{f}\right)>0$ and so $x_{f} \in \mathscr{N}_{0}$. 
It follows now from condition (ii) of Theorem III.2 by applying $f_{-1}$ on the inequality, that:

$$
\begin{aligned}
0 & <f_{-1}\left(2 M_{1}\left(x_{f}\right)\right)=f_{-1}\left(2 M_{1}\left(\left(x_{f}\right)^{*}\right)\right) \\
& \leqq f_{-1}\left(2 M_{-1}\left(\left(x_{f}\right)^{*}\right)\right)=\frac{m_{-1}\left(\left(x_{f}\right)^{*}\right)}{m_{0}\left(\left(x_{f}\right)^{*}\right)}=0,
\end{aligned}
$$

which is a contradiction, proving the first statement of the Lemma.

To prove the other statements of the Lemma, compute with the notations of above

$$
m_{k}\left(x_{f}\right)=\int_{\mathbb{R}} \lambda^{k}|\hat{f}(\lambda)|^{2} d\left\|E_{\lambda} x \Omega\right\|^{2}
$$

$k=0,1,2, \ldots$ If (i) or (ii) of Theorem III.3 are satisfied, then $m_{2 n+1}(x)$ is positive on $\mathscr{N}_{0}$ and the expression (13) implies that the spectral measure $d\left\|E_{\lambda} x \Omega\right\|^{2}$ has its support in $\mathbb{R}^{+}$. Take some $\lambda_{0}$ in this support. Choose a sequence of functions $\left(\hat{f}_{\ell}\right)_{\ell \in \mathbb{N}}$ in $\mathscr{D}$ such that

$$
\text { support } \left.\hat{f}_{\ell}=\left[\lambda_{0}-\frac{1}{\ell}, \lambda_{0}+\frac{1}{\ell}\right] \text { and } \hat{f}_{\ell}(\lambda)>0 \quad \text { for } \quad \lambda \in\right] \lambda_{0}-\frac{1}{\ell}, \lambda_{0}+\frac{1}{\ell}[\text {, }
$$

then for $k=0,1,2, \ldots$

$$
\lim _{\ell \rightarrow \infty} \frac{m_{k}\left(x_{f_{\ell}}\right)}{m_{0}\left(x_{f_{\ell}}\right)}=\lambda_{0}^{k}
$$

From the inequalities (i) or (ii) of Theorem III.3

Hence

$$
M_{2 n-1}\left(x_{f_{\ell}}\right) \leqq M_{2 n}\left(x_{f_{\ell}}\right) .
$$

$$
M_{2 n-1}\left(x_{f_{\ell}}\right)^{2 n-1} \text { th } M_{2 n-1}\left(x_{f_{\ell}}\right) \leqq M_{2 n}\left(x_{f_{\ell}}\right)^{2 n-1} \text { th } M_{2 n}\left(x_{f_{\ell}}\right) .
$$

However by (8) and (14), the left hand side of this inequality tends as $\ell \rightarrow \infty$

$$
\lim _{\ell} \frac{1}{2^{2 n-1}} \frac{m_{2 n-1}\left(x_{f_{\ell}}\right)}{m_{0}\left(x_{f_{\ell}}\right)}=\left(\frac{\lambda_{0}}{2}\right)^{2 n-1}
$$

and for the right hand side

$$
\lim _{\ell} f_{2 n}\left(2 M_{2 n}\left(x_{f_{\ell}}\right)\right)=\lim _{\ell} \frac{m_{2 n}\left(x_{f_{\ell}}\right)}{m_{0}\left(x_{f_{\ell}}\right)}=\lambda_{0}^{2 n}
$$

After substitution we get

$$
\left(\frac{\lambda_{0}}{2}\right)^{2 n-1} \leqq\left(\frac{\lambda_{0}}{2}\right)^{2 n-1} \operatorname{th} \frac{\lambda_{0}}{2}
$$

implying that $\lambda_{0}=0$ or the support of the measure $d\left\|E_{\lambda} x \Omega\right\|^{2}$ is reduced to zero, hence $H \times \Omega=0$.

Therefore, if $t_{1}, t_{2} \in \mathbb{R}$ then $\omega\left(x^{*} x\right)=\omega\left(\alpha_{t_{1}}\left(x^{*}\right) \alpha_{t_{2}}(x)\right)>0$.

Let $\mathscr{M}_{t}$ be the mean over $\mathbb{R}$, define the map $\Phi$ of $\mathscr{M}$ in $\mathscr{M}$ by 


$$
\mathscr{M}_{t}\left(\xi, \alpha_{t}(x) \eta\right)=(\xi, \Phi(x) \eta) ; \quad \xi, \eta \in \mathscr{H}
$$

then $\Phi(x) \in \mathscr{M}_{\alpha}$, and as $\mathscr{M}_{\alpha}$ is abelian

$$
\begin{aligned}
\omega\left(x^{*} x\right) & =\omega\left(\Phi(x)^{*} \Phi(x)\right) \\
& =\omega\left(\Phi(x) \Phi\left(x^{*}\right)\right)=\mathscr{M}_{t} \omega\left(\Phi(x) \alpha_{t}\left(x^{*}\right)\right)=0,
\end{aligned}
$$

as $\alpha_{t}\left(x^{*}\right) \Omega=0$.

Proof of Theorem III.2. The proof that (i) implies the KMS-property is given in Lemma III.4. Now we proceed to the proof that condition (ii) of Theorem III.2 implies the KMS-property. From Lemma III.1 the state $\omega$ is again time invariant and we consider again $\left\{E_{\lambda}\right\}_{\lambda}$, the spectral resolution of the Hamiltonian as in Lemma III.5.

For any $x \neq 0, x \in \mathscr{M}$, denote

$$
\begin{gathered}
d \mu_{x}(\lambda)=d\left\|E_{\lambda} x \Omega\right\|^{2}, \\
d v_{x}(\lambda)=d\left\|E_{\lambda} x^{*} \Omega\right\|^{2},
\end{gathered}
$$

and for $\hat{f} \in \mathscr{D}$

$$
\begin{aligned}
& \mu_{x}(f)=\int|\hat{f}(\lambda)|^{2} d \mu_{x}(\lambda), \\
& v_{x}(f)=\int|\hat{f}(\lambda)|^{2} d v_{x}(-\lambda) .
\end{aligned}
$$

Let $\hat{f} \in \mathscr{D}$ such that $x_{f} \neq 0$, then from Lemma III.5 $m_{0}\left(x_{f}\right)>0$ and from condition (ii) with $x=x_{f}$, respectively $\left(x_{f}\right)^{*}$, we get

$$
\begin{aligned}
& F\left(2 \int|\hat{f}(\lambda)|^{2} \frac{\left(1-e^{-\lambda}\right)}{\lambda} \frac{d \mu_{x}(\lambda)}{m_{0}\left(x_{f}\right)}\right) \leqq \frac{1}{2} \int \lambda|\hat{f}(\lambda)|^{2} \frac{\left[d \mu_{x}(\lambda)-d v_{x}(-\lambda)\right]}{m_{0}\left(x_{f}\right)}, \\
& F\left(2 \int|\hat{f}(\lambda)|^{2} \frac{\left(e^{\lambda}-1\right)}{\lambda} \frac{d v_{x}(-\lambda)}{m_{0}\left(x_{f}\right)}\right) \leqq \frac{1}{2} \int \lambda|\hat{f}(\lambda)|^{2} \frac{\left[d \mu_{x}(\lambda)-d v_{x}(-\lambda)\right]}{m_{0}\left(x_{f}\right)} .
\end{aligned}
$$

Also from Lemma III.5 support $\mu_{x}=-$ support $v_{x} \neq \phi$; take $\lambda_{0} \in$ support $\mu_{x}$ and a sequence $\left(\hat{f}_{\ell}\right)_{\ell \in \mathbb{N}}$ in $\mathscr{D}$ such that

$$
\text { support } \left.\hat{f}_{\ell}=\left[\lambda_{0}-\frac{1}{\ell}, \lambda_{0}+\frac{1}{\ell}\right] \text { and } \hat{f}_{\ell}(\lambda)>0 \quad \text { for } \lambda \in\right] \lambda_{0}-\frac{1}{\ell}, \lambda_{0}+\frac{1}{\ell}[\text {. }
$$

Using the convexity of the function $F$ (see Lemma II.3) we sum up the inequalities (15) and (16) with the weights respectively

to get:

$$
\frac{e^{\lambda_{0}}}{e^{\lambda_{0}}+1} \text { and } \frac{1}{e^{\lambda_{0}}+1}
$$

$$
\begin{aligned}
& F\left(2 \int \frac{\left|\hat{f}_{\ell}(\lambda)\right|^{2}}{\left(e^{\lambda_{0}}+1\right) m_{0}\left(x_{f_{\ell}}\right)}\left[\frac{e^{\lambda_{0}}\left(1-e^{-\lambda}\right)}{\lambda} d \mu_{x}(\lambda)+\frac{\left(e^{\lambda}-1\right)}{\lambda} d v_{x}(-\lambda)\right]\right) \\
& \quad \leqq \frac{1}{2} \int \lambda\left|\hat{f}_{\ell}(\lambda)\right|^{2} \frac{\left[d \mu_{x}(\lambda)-d v_{x}(-\lambda)\right]}{m_{0}\left(x_{f_{\ell}}\right)} .
\end{aligned}
$$


Taking lim inf (lim) of both sides of (17), we get by the mean value theorem

$$
\frac{\lambda_{0}}{2} \text { th } \frac{\lambda_{0}}{2} \leqq \lim \frac{\lambda_{0}}{2} \frac{\frac{\mu_{x}\left(f_{\ell}\right)}{v_{x}\left(f_{\ell}\right)}-1}{\frac{\mu_{x}\left(f_{\ell}\right)}{v_{x}\left(f_{\ell}\right)}+1} .
$$

Suppose now $\lambda_{0}>0$ (the case $\lambda_{0}<0$ can be treated in the same way), then from (18)

$$
e^{\lambda_{0}} \leqq \lim \frac{\mu_{x}\left(f_{\ell}\right)}{v_{x}\left(f_{\ell}\right)}
$$

Take now $\overline{\lim }$ of (16). Observe that the function $F$ is decreasing and use inequality (19) to get subsequently:

$$
\begin{aligned}
\frac{\lambda_{0}}{2} \overline{\lim \frac{\mu_{x}\left(f_{\ell}\right)}{v_{x}\left(f_{\ell}\right)}-1} \frac{\mu_{\ell}\left(f_{\ell}\right)}{v_{x}\left(f_{\ell}\right)}+1 & \geqq F\left(2 \frac{e^{\lambda_{0}}-1}{\lambda_{0}} \frac{\lim }{\frac{\mu_{x}\left(f_{\ell}\right)}{v_{x}\left(f_{\ell}\right)}+1}\right) \\
& \geqq F\left(\frac{\frac{\mu_{x}\left(f_{\ell}\right)}{v_{x}\left(f_{\ell}\right)}-1}{\frac{1}{2} \ln \frac{\mu_{x}\left(f_{\ell}\right)}{v_{x}\left(f_{\ell}\right)}} \frac{\lim }{\frac{\mu_{x}\left(f_{\ell}\right)}{v_{x}\left(f_{\ell}\right)}+1}\right) \\
& \geqq \varlimsup \frac{1}{2}\left(\ln \frac{\mu_{x}\left(f_{\ell}\right)}{v_{x}\left(f_{\ell}\right)}\right) \frac{\frac{\mu_{x}\left(f_{\ell}\right)}{v_{x}\left(f_{\ell}\right)}-1}{\frac{\mu_{x}\left(f_{\ell}\right)}{v_{x}\left(f_{\ell}\right)}+1} .
\end{aligned}
$$

Hence

$$
\frac{\lambda_{0}}{2} \geqq \frac{1}{2} \ln \varlimsup \frac{\mu_{x}\left(f_{\ell}\right)}{v_{x}\left(f_{\ell}\right)},
$$

and together with (19):

$$
e^{\lambda_{0}}=\lim _{\ell \rightarrow \infty} \frac{\mu_{x}\left(f_{\ell}\right)}{v_{x}\left(f_{\ell}\right)} .
$$

The case $\lambda_{0}=0$ yields immediately from (15) and (16) that

Hence

$$
\lim _{\ell \rightarrow \infty} \frac{\mu_{x}\left(f_{\ell}\right)}{v_{x}\left(f_{\ell}\right)}=1 .
$$

$$
\frac{d \mu_{x}\left(\lambda_{0}\right)}{d v_{x}\left(-\lambda_{0}\right)}=\lim _{\ell \rightarrow \infty} \frac{\mu_{x}\left(f_{\ell}\right)}{v_{x}\left(f_{\ell}\right)}=e^{\lambda_{0}}
$$

for all $\lambda_{0} \in \operatorname{supp} \mu_{x}$. But this is well known to be equivalent to the KMScondition $[10]$. 
Proof of Theorem III.3. If the conditions of Theorem III.3 are satisfied, then the state $\omega$ is separating and we consider again for every $x \in \mathscr{M}, x \neq 0$ the measures $\mu_{x}(\lambda), v_{x}(\lambda)$ and the sequence of functions $\left(f_{\ell}\right)_{\ell \in \mathbb{N}}$, as in the proof of Theorem III.2. Consider first the situation that condition (i) is satisfied, then for $\lambda_{0}>0, \lambda_{0} \in$ support $\mu_{x}$

$$
\begin{aligned}
\lim _{\ell} M_{2 n}\left(x_{f_{\ell}}\right)^{2 n} & =\lim _{\ell} \int\left(\frac{\lambda}{2}\right)^{2 n}\left|\hat{f}_{\ell}(\lambda)\right|^{2} \frac{d \mu_{x}(\lambda)+d \nu_{x}(-\lambda)}{m_{0}\left(x_{f_{\ell}}\right)} \\
& =\left(\frac{\lambda_{0}}{2}\right)^{2 n} .
\end{aligned}
$$

As $M_{2 n-1}(x) \leqq M_{2 n}(x)$ :

$$
M_{2 n-1}\left(x_{f_{\ell}}\right)^{2 n-1} \text { th } M_{2 n-1}\left(x_{f_{\ell}}\right) \leqq M_{2 n}\left(x_{f_{\ell}}\right)^{2 n-1} \text { th } M_{2 n}\left(x_{f_{\ell}}\right) .
$$

Take $\overline{\lim }$ of this inequality and use $(21)$ to get:

$$
\begin{aligned}
\left(\frac{\lambda_{0}}{2}\right)^{2 n-1} \operatorname{th} \frac{\lambda_{0}}{2} & \geqq \varlimsup M_{2 n-1}\left(x_{f_{\ell}}\right)^{2 n-1} \operatorname{th} M_{2 n-1}\left(x_{f_{\ell}}\right) \\
& =\varlimsup \lim \int\left(\frac{\lambda}{2}\right)^{2 n-1}\left|\hat{f}_{\ell}(\lambda)\right|^{2} \frac{\left[d \mu_{x}(\lambda)-d v_{x}(-\lambda)\right]}{m_{0}\left(x_{f_{\ell}}\right)} \\
& =\left(\frac{\lambda_{0}}{2}\right)^{2 n-1} \overline{\lim } \frac{\frac{\mu_{x}\left(f_{\ell}\right)}{v_{x}\left(f_{\ell}\right)}-1}{\frac{\mu_{x}\left(f_{\ell}\right)}{v_{x}\left(f_{\ell}\right)}+1} .
\end{aligned}
$$

Hence

$$
\varlimsup \frac{\mu_{x}\left(f_{\ell}\right)}{v_{x}\left(f_{\ell}\right)} \leqq e^{\lambda_{0}}
$$

Analogously from $M_{2 n}(x) \leqq M_{2 n+1}(x)$ :

$$
e^{\lambda_{0}} \leqq \underline{\lim } \frac{\mu_{x}\left(f_{\ell}\right)}{v_{x}\left(f_{\ell}\right)}
$$

The same inequalities are deduced for $\lambda_{0}<0$ in a similar way, therefore

$$
\frac{d \mu_{x}(\lambda)}{d v_{x}(-\lambda)}=e^{\lambda} \quad \text { for } \lambda \neq 0 .
$$

Suppose now that the measure $\mu_{x}(\lambda)$ is atomic in $\lambda=0$.

Let $E_{0}$ be the projection on the invariant vectors and $\Phi$ the mean of $\alpha_{t}$, as in the proof of Lemma III.5, then using condition (12):

$$
\begin{aligned}
\left(\Omega, x^{*} E(0) x \Omega\right) & =\left(\Omega, \Phi\left(x^{*}\right) \Phi(x) \Omega\right) \\
& =\left(\Omega, \Phi(x), \Phi\left(x^{*}\right) \Omega\right) \\
& =\left(\Omega, x E(0) x^{*} \Omega\right),
\end{aligned}
$$

proving that (22) holds for $\lambda=0$. 
Suppose now that condition (ii) is satisfied, i.e. for all $x \in \mathscr{M}$

$$
M_{2 n}\left(x_{f_{\ell}}\right) \leqq M_{2 n+1}\left(x_{f_{\ell}}\right) \leqq M_{2 n+2}\left(x_{f_{\ell}}\right) .
$$

From (21) for $\lambda_{0} \in$ support $\mu_{x}$ :

Therefore

$$
\lim _{\ell} M_{2 n+1}\left(x_{f_{\ell}}\right)=\frac{\lambda_{0}}{2} .
$$

Hence for $\lambda_{0} \neq 0$

$$
\begin{aligned}
\left(\frac{\lambda_{0}}{2}\right)^{2 n+1} \text { th } \frac{\lambda_{0}}{2} & =\lim _{\ell} M_{2 n+1}\left(x_{f_{\ell}}\right)^{2 n+1} \text { th } M_{2 n+1}\left(x_{f_{\ell}}\right) \\
& =\left(\frac{\lambda_{0}}{2}\right)^{2 n+1} \lim _{\ell} \frac{\frac{\mu_{x}\left(f_{\ell}\right)}{v_{x}\left(f_{\ell}\right)}-1}{\frac{\mu_{x}\left(f_{\ell}\right)}{v_{x}\left(f_{\ell}\right)}+1} .
\end{aligned}
$$

$$
\frac{d \mu_{x}\left(\lambda_{0}\right)}{d v_{x}\left(-\lambda_{0}\right)}=e^{\lambda_{0}} \text {. }
$$

The case $\lambda_{0}=0$ is treated as above.

As it was remarked above, Theorems III.2 and 3 constitute an infinite set of correlation inequalities which are strong enough to impose equilibrium on the system. As far as the first condition is concerned, it is known that it can be written in a form [12] which gives an easy interpretation of the inequality in terms of a notion of stability [13]. It is evident that it would be instructive to look for the appropriate notions of stability corresponding to the other set of inequalities.

Another aspect of the result which we want to stress is that we give the characterization of KMS-states by means of maximum three powers of the generator of the time evolution.

\section{References}

1. Kubo, R.: J. Phys. Soc. Japan 12, 570 (1957)

2. Mori, H.: Progr. Theor. Phys. 33, 423 (1965)

3. Naudts, J., Verbeure, A., Weder, R.: Commun. Math. Phys. 44, 87 (1975)

4. Verbeure, A., Weder, R.: Commun. Math. Phys. 44, 101 (1975)

5. Dupuis, M.: Progr. Theor. Phys. 37, 502 (1966)

6. Dyson, F.J., Lieb, E.H., Simon, B.: J. Stat. Phys. 18, 335 (1978)

7. Falk, H., Bruch, L.: Phys. Rev. 180, 442 (1969)

8. Martens, R., Verbeure, A.: Lett. Math. Phys. 3, 413 (1979)

9. Roepstorff, G.: Commun. Math. Phys. 46, 253 (1976)

10. Haag, R., Kastler, D., Trych-Pohlmeyer, E.B.: Commun. Math. Phys. 38, 173 (1974)

11. Pusz, W., Woronowicz, S.L.: Commun. Math. Phys. 58, 273 (1978)

12. Fannes, M., Verbeure, A.: Commun. Math. Phys. 57, 165 (1977)

13. Fannes, M., Verbeure, A.: J. Math. Phys. 19, 558 (1978)

Communicated by H. Araki

Received February 21, 1981 
